\title{
Can HLA type I and II alleles presence be associated with the clinical spectrum of CHIKV infection?
}

\author{
Juan C. Rueda ${ }^{1}$, Ana M. Santos ${ }^{2}$, Jose-Ignacio Angarita ${ }^{2}$, Eugenia-Lucia Saldarriaga $^{2}$, \\ Ingris Peláez-Ballestas ${ }^{3}$, Alejandro Silva Espinosa ${ }^{4}$, Ignacio Briceño-Balcázar ${ }^{4}$, Sofia \\ Arias-Correal ${ }^{2}$, José Arias Correal ${ }^{2}$, Catalina Villota-Erazo ${ }^{2}$, Viviana Reyes ${ }^{2}$, Santiago \\ Bernal-Macías $^{2}$, Mario H. Cardiel ${ }^{5}$, and John Londono ${ }^{2}$ \\ ${ }^{1}$ Student from the Biosciences Programme Faculty of Medicine and Engineering \\ Universidad de La Sabana Chía Colombia \\ ${ }^{2}$ Grupo de Espondiloartropatías Rheumatology Department Universidad de La Sabana \\ Chía Colombia \\ ${ }^{3}$ Hospital General de Mexico Dr Eduardo Liceaga \\ ${ }^{4}$ Grupo de Genética Humana Universidad de La Sabana Chía Colombia \\ ${ }^{5}$ Centro de Investigación Clínica de Morelia SC Morelia Mexico
}

May 12, 2021

\begin{abstract}
Host immune response as well as virulence factors are key in disease susceptibility. There are no known association studies of HLA class I and II alleles with chikungunya (CHIKV) infection in Latin American population. We aim to identify Human Leukocyte Antigen (HLA) alleles present in patients with CHIKV infection when compared to healthy controls, as well as allele association with the clinical spectrum of the disease. A cross-sectional analysis nested in a community cohort was carried out. We included patients 18 years and older with serological confirmation of CHIKV infection. HLA typing of HLA-A, HLA-B and HLA-DRB1 alleles was performed. Two-by-two tables were used to establish associations between allele presence and clinical characteristics. Data from 65 patients with confirmed CHIKV infection were analyzed for HLA typing. CHIKV infection was associated with the presence of HLA-A*68, HLA-B*35, HLA-DRB*01, HLA-DRB1*04 and HLA-DRB1*13 alleles with statistical significance when compared to healthy subjects. A statistically significant relationship was found between the presence of rash in the face or the abdomen and the presence of HLA-DRB $1 * 04$. Our study demonstrated that in our cohort, HLA type I as well as type II alleles are associated with CHIKV infection, and specifically an HLA type II allele with dermatological symptoms. Further research is needed to set a path for future investigation on genes outside the HLA system to improve knowledge in the pathophysiology of CHIKV infection and its host-pathogen interaction.
\end{abstract}

\section{Hosted file}

Manuscript HLA and CHIKV.pdf available at https://authorea.com/users/413540/articles/521826can-hla-type-i-and-ii-alleles-presence-be-associated-with-the-clinical-spectrum-ofchikv-infection 